\title{
Comparative study of highly dense aluminium- and gallium-doped zinc oxide transparent conducting sol-gel thin films
}

\author{
NAJI AL DAHOUDI \\ Physics Department, Al Azhar University - Gaza, P.O. Box 1277, Gaza, Palestine
}

MS received 30 September 2013; revised 18 December 2013

\begin{abstract}
Transparent conducting aluminium- and gallium-doped zinc oxide (AZO and GZO) layers have been deposited by spin coating on glass substrates. The coatings have been sintered in air at $450{ }^{\circ} \mathrm{C}$ for $30 \mathrm{~min}$ and then post-annealed at $350{ }^{\circ} \mathrm{C}$ in a reducing atmosphere for $30 \mathrm{~min}$. The electrical, optical and morphological properties of both coatings have been studied and compared. The conventional sols lead to very thin coating, typically $24 \mathrm{~nm}$ for a single layer of AZO and $32 \mathrm{~nm}$ of GZO with electrical resistivity of 0.72 and $0.35 \Omega \mathrm{cm}$, respectively. The value however, drastically decreases down to a minimum of $2.6 \times 10^{-2} \Omega \mathrm{cm}$ for AZO and $1.76 \times 10^{-2} \Omega \mathrm{cm}$ for GZO, when five multilayer coatings are made. The origin of these differences is due to the different morphology of the coatings showing different electron scattering process. The GZO sol leads to denser smoother structure (porosity of $5 \%$ ) layers with an average roughness of $2.76 \AA$, while the AZO coating is formed by a more porous assembly (porosity of $20 \%$ ) with an average roughness of $3.46 \AA$. Both coatings exhibit high transparency $(T>85 \%)$ in the visible spectrum range with a slight shift of the absorption energy gap.
\end{abstract}

Keywords. AZO; GZO; transparent conductive film; electrical and optical properties.

\section{Introduction}

Wide band gap $\left(E_{\mathrm{g}} \geq 3 \mathrm{eV}\right) n$-type semiconductors as tin-doped indium oxide (ITO), antimony-doped tin oxide (ATO), fluorine-doped tin oxide (FTO) or aluminiumdoped zinc oxide (AZO) are among the most important used transparent conducting materials (Coutts et al 2000; Gordon 2000; Berry et al 2011). Such materials are transparent in the visible range and electrically conductive at the same time, which put them today as valuable for many applications, such as electrodes in optoelectronic devices, as IR reflecting or heatable layers, for electromagnetic shielding or for dissipating static charging (Hartnagel et al 1995; Lewis and Paine 2000). The values of the electrical sheet resistance of such materials are needed to match the industrial applications. Some applications require a sheet resistance in the range between $1 \mathrm{k} \Omega_{\square}$ and $1 \mathrm{M} \Omega_{\square}$, like electrostatic and antistatic applications (Texter and Lelental 1999; Al Dahoudi et al 2001; Augulis et al 2005), however, other applications need lower sheet resistance in the range of $1-100 \Omega_{\square}$, such as for optoelectronics devices (Fernando et al 2007; Özgür et al 2010; Godlewski et al 2011).

Till today, ITO is the most interesting high performance material that has been commercialized and used for

(naji@alazhar.edu.ps) wide range of applications, however, the exhaustion of indium source make its price increasing every day. On the other hand, zinc oxide-based materials may be considered as promising substitutes for ITO layers, because of their optoelectronic properties in addition to low cost, high stability and excellent surface uniformity (low roughness). Doping zinc oxide with some elements like aluminium, gallium, boron, niobium and indium has a great impact for having enhancements of its optical and electrical properties. Aluminium and gallium doping are considered as a favourable doping of zinc oxide, which generate extra electrons making them useful for transparent conducting layers application.

Furthermore, many deposition techniques were already used for obtaining transparent conducting oxide (TCO) films on glass substrates (Hong and $\mathrm{Xu}$ 2010; Gong et al 2011). Although, the electrical resistivity of the films produced via the complicated and expensive physical deposition techniques is still lower than those produced by wet chemical deposition routes, sol-gel technique is one of the attractive utilized methods due to its low cost, simplicity and easy to be controlled.

In this paper, a wet chemical deposition route was used to deposit AZO and GZO to produce transparent conducting layers via spin coating on glass substrates. The structural, optical and electrical properties of AZO and GZO films are characterized and a comparative study between AZO and GZO was carried out. 


\section{Experimental}

\subsection{AZO and GZO sols}

The AZO and GZO sols were synthesized by dissolving $1.362 \mathrm{~g}$ of zinc acetate dehydrate in $25 \mathrm{~mL}$ of 2-isopropoxy ethanol with continuous stirring. $0.4 \mathrm{~g}$ of diethanol amine (DEA) is added gradually, till clear solution is obtained. In the case of AZO, $0.025 \mathrm{~g}$ of aluminium isopropoxide is dissolved in a $0.25 \mathrm{~g}$ of DEA and then added to the zinc acetate dehydrate solution, where $\mathrm{Al} / \mathrm{Zn}=1 \mathrm{~mol} \%$. In the case of GZO, $0.02 \mathrm{~g}$ of gallium nitrate is dissolved in a $0.25 \mathrm{~g}$ of DEA and then added to the zinc acetate dehydrate solution, where $\mathrm{Ga} / \mathrm{Zn}=1 \mathrm{~mol} \%$. The resulted solution is left stirring for $2 \mathrm{~h}$ and kept aging for $24 \mathrm{~h}$ before coating.

The coating sol was deposited on borosilicate glasses using the spin-coating method. The coatings were then sintered in air at $450{ }^{\circ} \mathrm{C}$ during $30 \mathrm{~min}$ and further postannealed in forming gas $\left(\mathrm{N}_{2} / \mathrm{H}_{2}: 95 / 5\right)$ at $350{ }^{\circ} \mathrm{C}$ during $30 \mathrm{~min}$. Multilayers were made by repeating the whole coating procedure (deposition, sintering and annealing).

\subsection{Characterization}

The structural characterization of the deposited films was carried out using an X-ray diffractometer (XRD) (XPERT-PRO-MPD, Panalytical), using $\mathrm{Cu}$ anode material operating at $40 \mathrm{kV}$ and $30 \mathrm{~mA}$ with wavelength $(\mathrm{K} \alpha$ : $1.541837 \AA, \mathrm{K} \beta: 1.392220 \AA$ ) and the beam radius of $240 \mathrm{~mm}$. The thickness $(t)$ and the refractive index of the coatings were determined using a spectroscopic ellipsometer (Woollam M-2000DI). The surface morphology has been analysed using scanning electron microscopy (SEM) (FEI Quanta 400 FEG was used in high vacuum mode) and a Zygo Newview 5000 white light interferometer, where the roughness was determined. The sheet resistance $\left(R_{\square}\right)$ was measured by a 4-point probe technique using the Napson model (RG-7s and RT-70). The resistivity was calculated from the relation, $\rho=R_{\square}$. The transmittance has been determined using a Varian Cary $5 \mathrm{E}$ spectrophotometer in the wavelength range of 300 $3000 \mathrm{~nm}$.

\section{Results and discussion}

To obtain thick film of sol-gel thin film, multilayer deposition is required, which is not adequate for industrial application. Figure 1 shows the thickness and the transmission of spin-coated AZO (figure 1a) and GZO (figure 1b) layers on glass substrate. The layers were sintered in air at $450{ }^{\circ} \mathrm{C}$ and followed by post-treatment under forming gas at $350^{\circ} \mathrm{C}$. The increase in the thickness by increasing the number of layers is accompanied by a deterioration of their transparency from $91 \%$ to $81 \%$ for
AZO layers by increasing the thickness from 24 to $95 \mathrm{~nm}$. GZO layers exhibited lower reduction of their transparency compared with the AZO layers, where 5-layered $(110 \mathrm{~nm})$ GZO film processes transparency of $85 \%$.

The densification of the transparent conducting layers is a crucial issue for enhancing the electrical properties. To get a better insight on this phenomenon, the porosity of the films was estimated by measuring the refractive index of the coatings using the following relation (Furusaki et al 1994)

$$
P(\%)=\left[1-\frac{n_{\mathrm{p}}^{2}-1}{n_{\mathrm{s}}^{2}-1}\right] \times 100
$$

where $P$ and $n_{\mathrm{p}}$ are the porosity (in \%) and the refractive index of the porous film material, respectively, and $n_{\mathrm{s}}$ the theoretical refractive index of the dense material $\left(n_{\mathrm{s}}=2 \cdot 01\right.$ at $\lambda=550 \mathrm{~nm}$ ).

Figure 2(a) and (b) shows the refractive index and the porosity of AZO and GZO layers as a function of number of deposited layers. The significance reduction of the
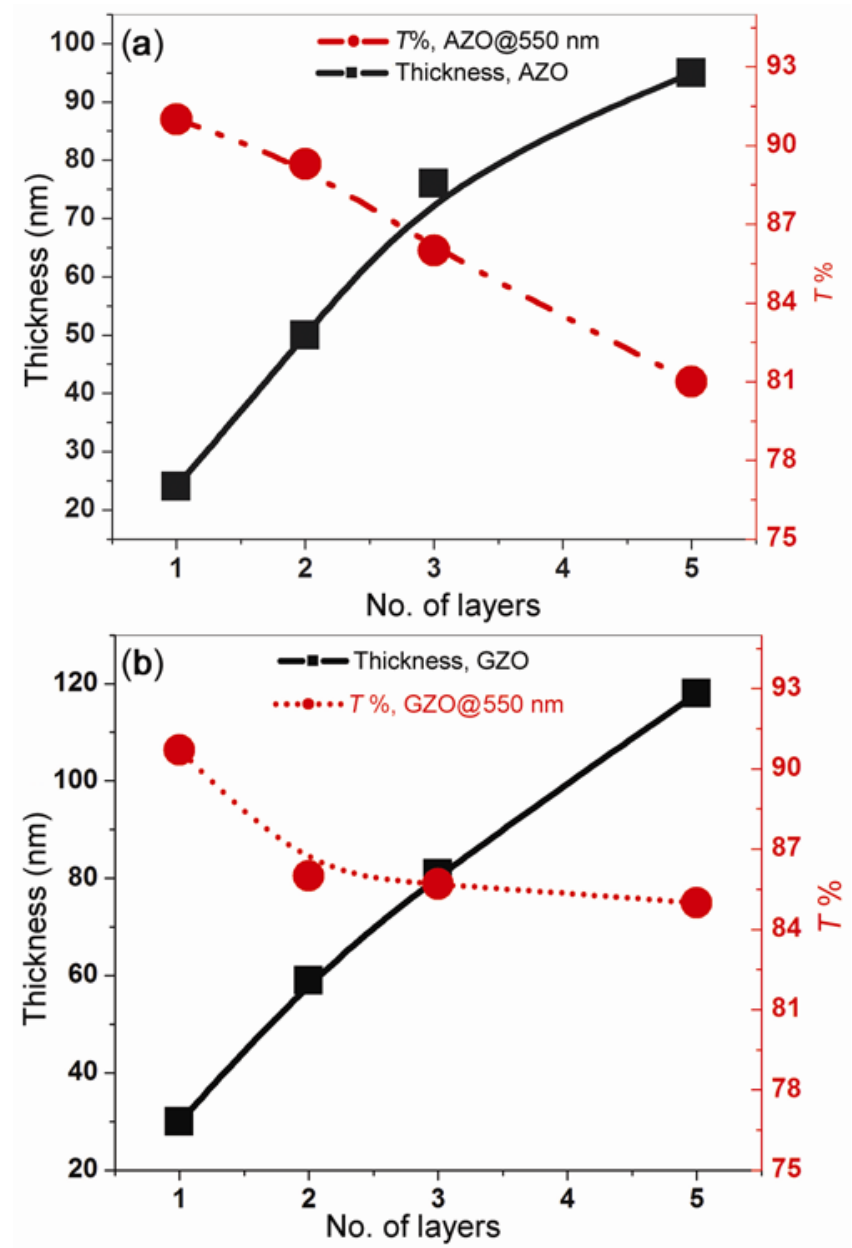

Figure 1. Thickness and transmission at $550 \mathrm{~nm}$ of spincoated AZO (a) and GZO (b) layers on glass substrates as a function of the number of deposited layers. 

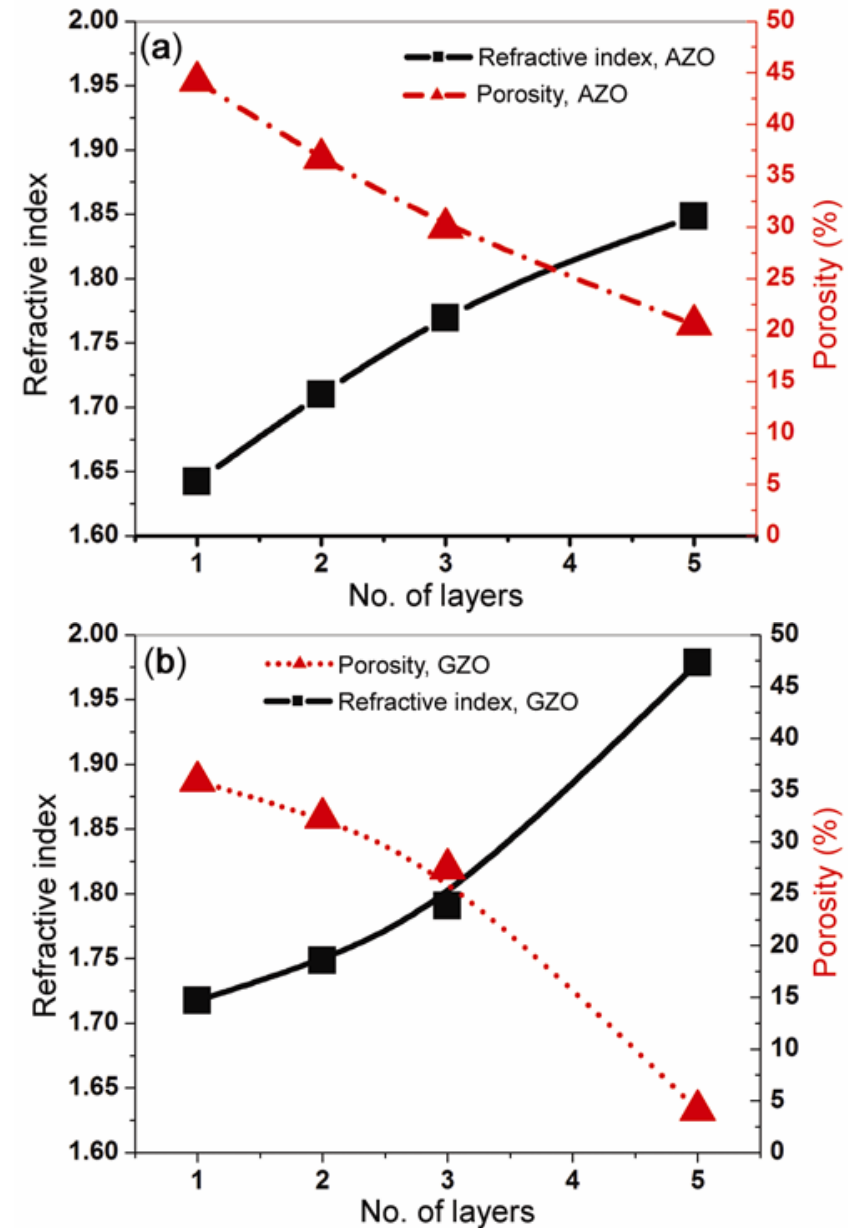

Figure 2. The refractive index and the porosity of AZO (a) and GZO (b) layers as a function of the number of deposited layers fired in air at $450{ }^{\circ} \mathrm{C}$.

porosity for GZO layers from $35 \%$ to $5 \%$ when the number of layers rises from 1 to 5 . Similar tendency is seen for AZO layers, however, AZO multi-layers are still more porous than $\mathrm{GZO}$ ones, where its porosity decreases from $45 \%$ to $21 \%$. It is expected that single layer is still porous and adding another layers results in filling the pores forming more dense morphology (Goebbert et al 2000). This means that firing the layers at relative low temperature $\left(450{ }^{\circ} \mathrm{C}\right)$ is enough to obtain dense film for both coatings.

\section{$3.1 X R D$}

Figure 3 exhibits the XRD pattern of AZO and GZO layers on glass substrate fired in air at $450{ }^{\circ} \mathrm{C}$ in addition to post-treatment at $350{ }^{\circ} \mathrm{C}$ in forming the gas atmosphere. The XRD pattern shows a zincite $\mathrm{ZnO}$ (wurtzite hexagonal structure, pdf no. 01-074-9940), which is the only detected crystallographic phase for both AZO and GZO layers. The crystallite size calculated for the $\left(\begin{array}{lll}1 & 0 & 0\end{array}\right)$,

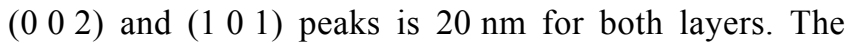

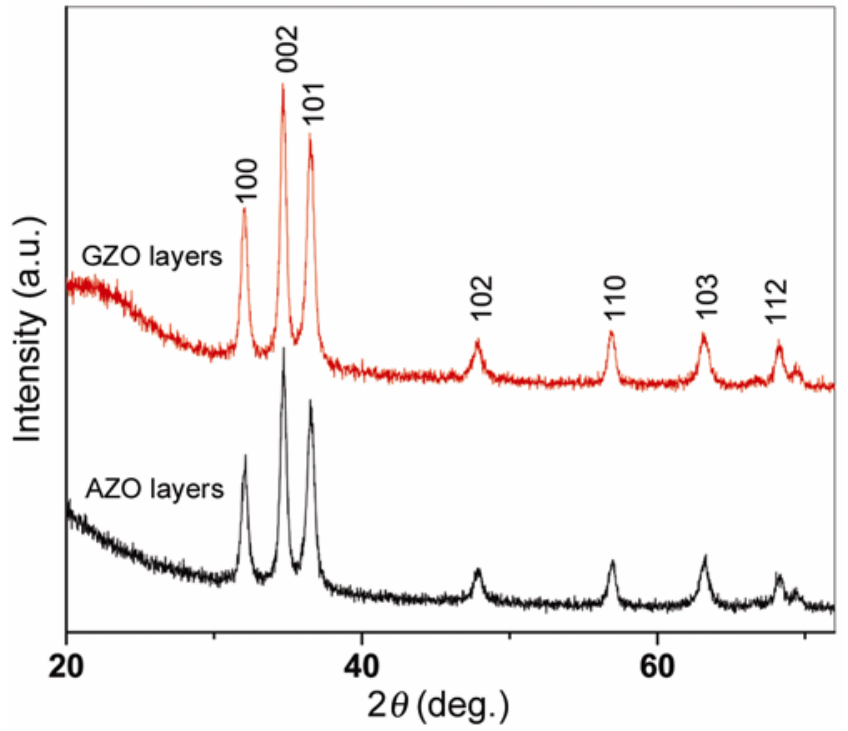

Figure 3. XRD patterns of AZO and GZO layers spin-coated on glass substrate fired in air at $450{ }^{\circ} \mathrm{C}$ and post-treated at $350{ }^{\circ} \mathrm{C}$ in forming the gas atmosphere.

textured coefficient, (llll $\left.\begin{array}{lll}1 & 0 & 1\end{array}\right) /\left(\begin{array}{lll}0 & 0 & 2\end{array}\right)$, for AZO sol-gel layers $(0.725)$ is lower than that of GZO layers $(0.798)$. This shows that GZO layer has stronger preferred growth along the $\left(\begin{array}{lll}0 & 0 & 2\end{array}\right)$ plane ( $c$-axis) than that of the AZO layers. The higher the $c$-axis preferred orientation is, the lower the electrical resistivity due to the reduction in the scattering of the carriers at the grain boundaries (Ohyama et al 1998; Musat et al 2004) which is confirmed by the results of the electrical properties of $A Z O$ and GZO layers.

\section{$3.2 S E M$}

Figure 4(a) and (b) shows SEM photographs for the surface of 5-layered AZO (a) and GZO (b) films sintered at $450{ }^{\circ} \mathrm{C}$ in air for $30 \mathrm{~min}$ and then post-annealed in forming gas at $350{ }^{\circ} \mathrm{C}$ for $30 \mathrm{~min}$. The microstructure exhibits densely-packed crystallites with grain size range of 40 $70 \mathrm{~nm}$ for GZO coatings and $30-50 \mathrm{~nm}$ for AZO coatings, which is larger than the thickness of at least 2 layers which indicates the absence of intergranular voids. This may enhance the mobility of the charge carriers, as a result of eliminating the discontinuities between the nanoparticles. This microstructure confirms the high density and the low porosity of the coated materials calculated in figure 2, which probably play an important role in enhancing the electrical conductivity. The densification of the coatings results in a filling of the open pores and the distance between the GZO and AZO grains become smaller, although the coatings sintered at relative low temperatures $\left(450^{\circ} \mathrm{C}\right)$. Choi and Lee (2001) reported that the film density influences the electrical properties of sputtered ITO thin films 

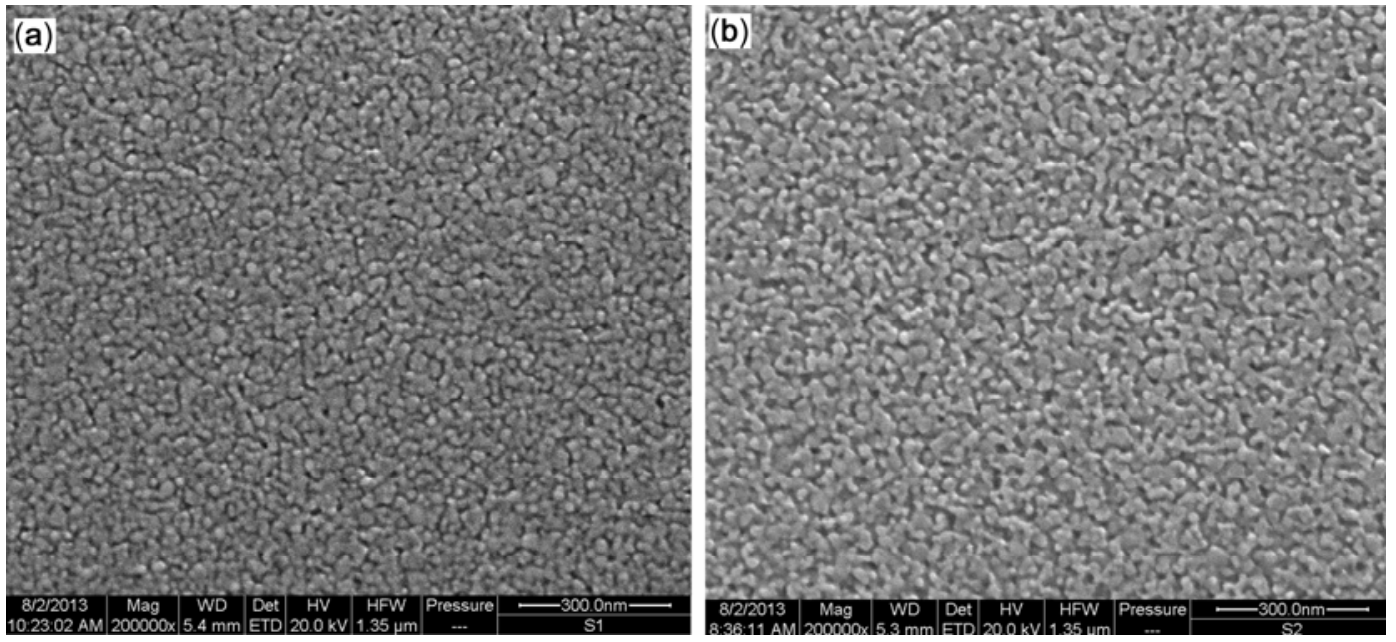

Figure 4. SEM images of 5-layered AZO (a) and GZO (b) coatings on glass substrates heated in air at $450{ }^{\circ} \mathrm{C}$ and followed by post-annealing in forming the gas.
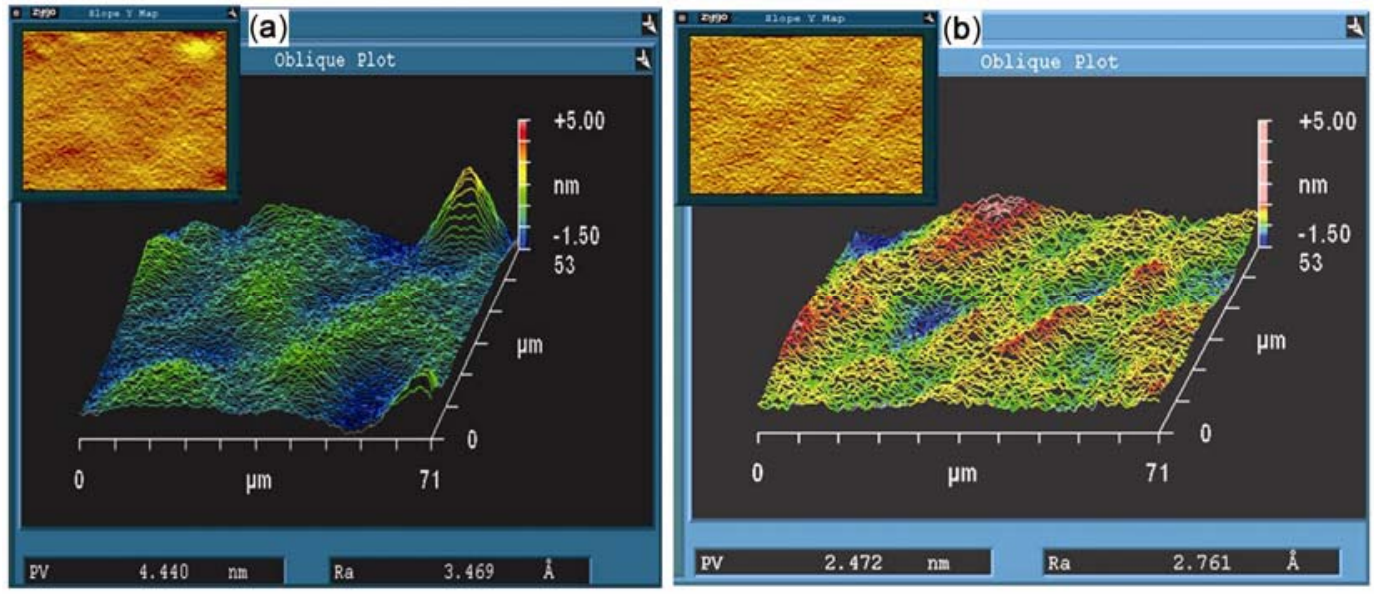

Figure 5. Surface morphology of 5-layered AZO (a) and GZO (b) coatings observed with WLI on a $53 \times 70 \mu \mathrm{m}^{2}$ area.

and in particular that the carrier mobility increases with the film density.

\subsection{White light interferometry (WLI)}

Figure 5 shows the topography of the surface of 5-layered AZO and GZO coatings measured using the white light interferometer. The coating roughness of a $53 \times 70 \mu \mathrm{m}^{2}$ area with a lateral resolution of $600 \mathrm{~nm}$ determined without electronic filtering is $R_{\mathrm{a}}=0.276 \mathrm{~nm}$ for GZO coatings, and a peak-to-valley maximum value of $R_{\mathrm{PV}}=2.47 \mathrm{~nm}$. For AZO coatings, an average roughness of $0.347 \mathrm{~nm}$ and a peak-to-valley maximum value of $R_{\mathrm{PV}}=4.47 \mathrm{~nm}$ are obtained. The surface of GZO coatings is denser and smoother than AZO. In general, no defects were found on both the coatings and their surfaces appear very smooth on this length scale.

\section{$3.4 \quad$ Electrical properties}

The sheet resistance of AZO and GZO coatings on glass substrates are depicted in figure 6(a) as a function of the number of deposited layers. The sheet resistance of both AZO and GZO coatings decrease gradually by increasing the thickness of layers. GZO layers exhibited lower sheet resistance than AZO. This indicates that doping zinc oxide with gallium may create more effective stoichiometric state than that produced by using aluminium. The lowest obtained sheet resistance for GZO 5-layered film is $1.5 \mathrm{k} \Omega_{\square}$, and for AZO layers, it is $2.9 \mathrm{k} \Omega_{\square}$. The decrease in the electrical resistivity of the layer is decreasing and also by the increase of the film thickness as shown in figure 6(b), which is a result of the coalescing of grains of crystallite that make the mobility of the charge carriers larger. It is observed from figure 2 that the densification of the conducting layer is enhanced by reducing 

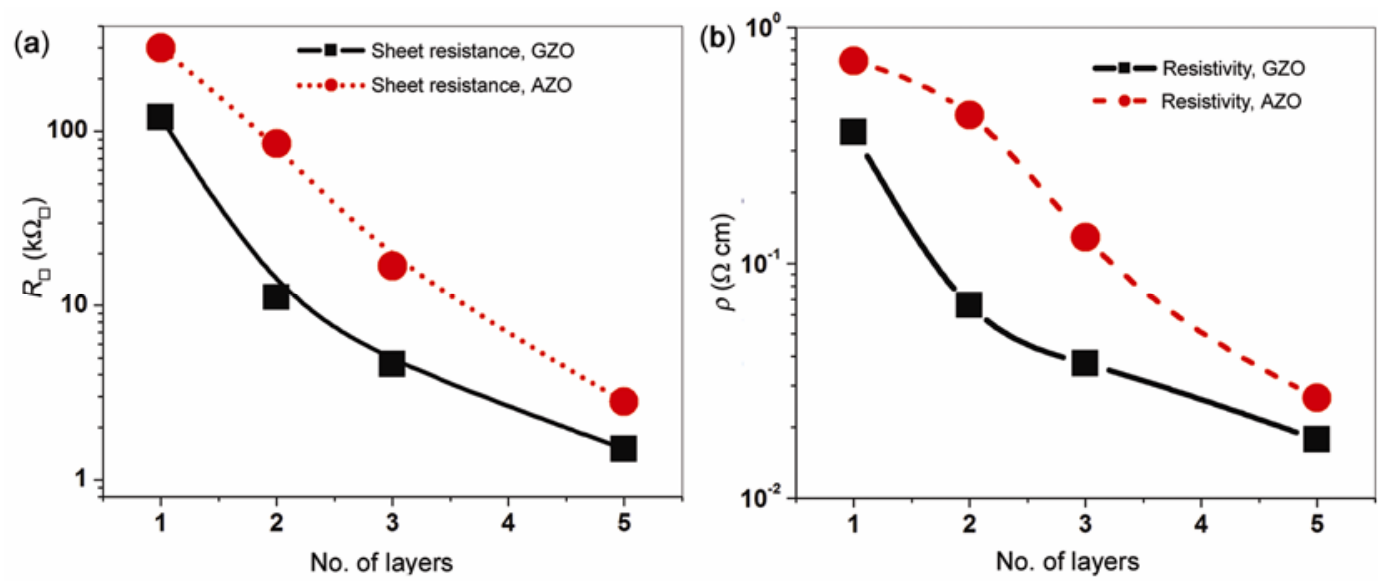

Figure 6. Sheet resistance (a) and resistivity (b) of GZO and AZO films as a function of the number of layers.
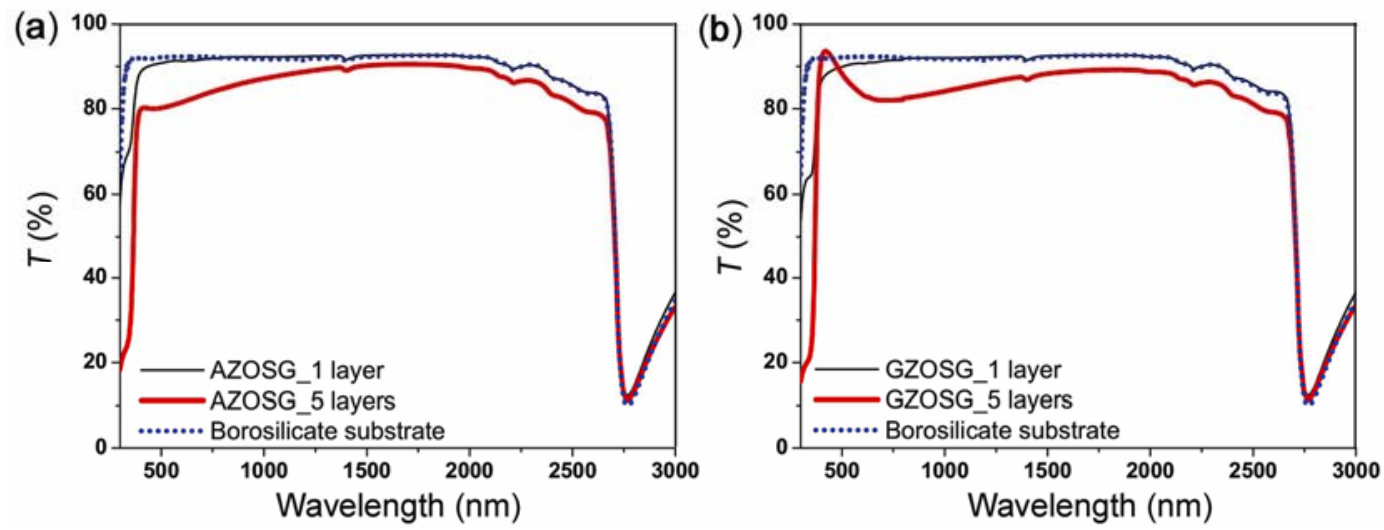

Figure 7. UV-Vis-NIR transmission spectra for 1- and 5-layered AZO films (a) and GZO films (b).

the porosity of the layers. This is confirmed referring to the SEM images shown in figure 4, where the particles emerged and diffused into each other forming an easy electron path and eliminate the existence of pores and voids, which are the scattering sources of the charge carriers in the layer.

The GZO layers have superior electrical conductivity $\left(\sigma=56 \Omega^{-1} \mathrm{~cm}^{-1}\right)$ compared with the AZO layers $(\sigma=$ $37 \Omega^{-1} \mathrm{~cm}^{-1}$ ), where the GZO films denser than AZO ones showing a minimal porosity of $\approx 5 \%$ less than that of $\mathrm{AZO} \approx 20 \%$. The surface of the GZO films is smoother than that of $\mathrm{AZO}$, which depicted from the average roughness shown in figure 5. GZO 5-layered film exhibits an average roughness of $0.27 \mathrm{~nm}(0.24 \%$ of the thickness $)$. The AZO 5-layered film exhibits an average roughness of $0.34 \mathrm{~nm}(0.46 \%$ of the thickness $)$. The densification of the film and its average roughness have a significant impact on the electrical mobility of the charge carriers, and hence, to the overall electrical conductivity.

\subsection{Optical properties}

Figure 7(a) and (b) shows the UV-Vis-NIR optical transmission of 1- and 5-layered AZO film (a) and GZO film (b). Both materials exhibit high transmission $>90 \%$ for one-layered film and $>85 \%$ for 5 -layered one, which is close to the transmission of the glass substrate. At the same time, a sharp decrease in the transmittance (strong absorption) at wavelength below $400 \mathrm{~nm}$, for both films. It is observed that the transmission edge of the AZO layers occurs at lower wavelength compared with the GZO. This narrowing of the band energy gap for GZO compared to AZO films may be explained as a result of an increase in the particle size and a decrease in the specific surface area of the particles, where GZO film showed denser structure and less voids compared with the AZO (Van Bommel et al 1999). Zhao et al (2006) have been addressed a correlation with the charge carriers concentration and the narrowing of the energy gap.

\section{Conclusions}

The morphological, electrical and optical properties of transparent conducting AZO and GZO layers, that have been deposited by spin coating on glass substrates, are studied and compared. Both coatings show a zincite hexagonal structure, where the textured coefficient for AZO layers $(0.725)$ is lower than that of GZO layers 
(0.798). This shows that GZO layer has stronger preferred growth along the $\left(\begin{array}{lll}0 & 0 & 2\end{array}\right)$ plane ( $c$-axis) than that of the AZO layers. The electrical resistivity of both the coatings decreases gradually by increasing the number of layers to reach $1.76 \times 10^{-2} \Omega \mathrm{cm}$ for GZO and $2.6 \times 10^{-2} \Omega \mathrm{cm}$ for AZO. The GZO layers exhibit higher density and lower roughness than AZO ones, which leads to less electron scattering and hence, higher electron mobility of the charge carriers. Both coatings exhibit high transparency $(T>85 \%)$ in the visible spectrum range with a narrower band energy gap for GZO layers.

\section{Acknowledgements}

I want to express my gratitude to Dr Peter Oliveira for hosting and supporting me during my summer visit at the Leibniz Institute of New Materials (INM), Saarbrücken, Germany. I am very grateful to Dr Marcus Koch for doing the SEM measurements, Mr Rudolf Karos for his XRD measurements and Mr Bruno Schäfer for the ellipsometry analysis.

\section{References}

Al Dahoudi N, Bisht H, Göbbert C, Krajweski T and Aegerter M 2001 Thin Solid Films 392299

Augulis L, Serzentas S, Prosycevas I, Tamulevicius S and Zabarskas V 2005 Mater. Sci. 11235
Berry J, Fortunato E, Medvedeva J and Shigesato Y 2011 MRS Proc. 1315

Choi S and Lee J 2001 J. Vac. Sci. Technol. A19 2043

Coutts T J, Young D L and Li X 2000 MRS Symp. Proc. 623199

Fernando S, Harris K, Gerein N and Brett M 2007 MRS Proc. 105647

Furusaki T, Takahashi J and Kodira K 1994 J. Ceram. Soc. Jpn (Int. Ed.) 102202

Godlewski M, Guziewicz E, Kopalko K, Luka G, Lukasiewicz M I, Krajewski T, Kwiatkowski B S and Gieratowska S 2011 Low Temp. Phys. 37235

Goebbert C, Gasparro G, Schuler T, Krajewski T and Aegerter M 2000 J. Sol-Gel Sci. Technol. 19435

Gong J, Zhang X, Pei Z, Sun C and Wen L 2011 J. Mater. Sci. Technol. 27393

Gordon R G 2000 MRS Bull. 2552

Hartnagel H L, Dawar A L, Jain A K and Jagadish C 1995 Semiconducting transparent thin films (Bristol: Institute of Physics Publishing)

Hong R and Xu S 2010 J. Mater. Sci. Technol. 26872

Lewis B G and Paine D C 2000 MRS Bull. 2522

Musat V, Teixeira B, Fortunato E, Monteiro R C and Vilarinho P 2004 Surf. Coat. Technol. 180-181 659

Ohyama M, Kozuka H and Yoko T 1998 J. Am. Ceram. Soc. 81 1622

Özgür Ü, Hofstetter D and Morkoc H 2010 Proc. IEEE 981255

Texter J and Lelental M 1999 Langmuir 15654

Van Bommel M J, Groen A, Van Hall H A M, Keur W C and Bernards T N M 1999 J. Mater. Sci. 344803

Zhao Q, Xu X Y, Song X F, Zhang X Z, Yu D P, Li C P and Guo L 2006 Appl. Phys. Lett. 88033102 